\title{
MODELS OF FLARING LOOPS
}

\author{
A. GORDON EMSLIE
}

Department of Physics, UAH, Huntsville, AL 35899, U.S.A.

\begin{abstract}
We review the somewhat questionable concept of an isolated flare loop and the various physical mechanisms believed to be responsible, to some degree, for energy transport within the loop structure. Observational evidence suggests a predominant role for high-energy electrons as an energy transport mechanism, and we explore the consequences of such a scenario in some detail, focusing on radiation signatures in the soft X-ray, hard X-ray, and EUV wavebands, as observed by recent satellite observatories. We find that the predictions of flare loop models are in fact in excellent agreement with these observations, reinforcing both the notion of the loop as a fundamental component of solar flares and the belief that electron acceleration is an integral part of the flare energy release process.
\end{abstract}

\section{The Concept of a Flare Loop}

Although the magnetic field topology and geometry in regions of flaring activity may be (of necessity?) complicated, there has nevertheless been considerable enthusiasm in recent years devoted to the modeling of the behavior of an isolated looplike structure in response to flare energy dissipation. Such flare 'loops' are defined by a bundle of magnetic field lines, considered to be rigid, and usually the region outside of this bundle is considered to be field-free. The material in the loop is treated, in most cases, as a single fluid free to move in one dimension only, i.e., along the field lines within the bundle. Energy (whether in the form of accelerated particles, heat, hydrodynamical kinetic energy, or waves) also flows along this single dimension, is converted into heat and mass motions, and is ultimately radiated away.

Convenient as this construct may be for theoretical modelers, there is substantial cause to question the assumptions inherent within it. First, the bundle of field lines under consideration is in all probability part of a more global field structure, and is highlighted against the background field only because of the preferential concentration of radiating material along the field lines of the 'loop'. This would not be a serious problem if the field lines were infinitely rigid, since energy flow across field lines (and, therefore, into or out of the bundle) is strongly suppressed, except in the case of radiative transport (see, e.g., the discussion of cross-field energy transport by maser-produced microwaves; Melrose and Dulk, 1982, 1984). However, such an assumption of rigid field lines neglects both the magnetohydrodynamic response of the atmosphere and the possibility of dynamic evolution of the field through further reconnection with adjacent field lines as the flare proceeds. Gas pressures developed as a result of flare heating can in fact reach values comparable to the pressure of the ambient magnetic field (Emslie, 1981a). We see, therefore, that the assumption of an isolated, rigid, unyielding, non-evolving field structure is a highly questionable one.

Second, a single-fluid treatment of the energized gas is probably inadequate. Many heating mechanisms (e.g., Joule heating, electron bombardment) heat primarily elec-

(C) 1989 Kluwer Academic Publishers. Printed in Belgium. 
trons, while some (e.g., ion-cyclotron waves) preferentially heat the ions. Electron-ion temperature equilibration times can be a substantial fraction of the duration of the impulsive phase of a flare, so that at least a two-fluid treatment is called for at such early times in the event. Shoub (1983) has shown that, even in the quiet Sun, there exist regions of steep temperature gradient (such as the chromosphere/corona transition region) in which the momentum distribution function for the particles is probably far from Maxwellian, demanding a kinetic treatment in order to correctly deduce radiative loss rates, thermal conductive fluxes, etc. The even steeper temperature gradients found in flares make this result even more pertinent (Karpen and DeVore, 1987).

Even accepting the assumptions of the model, the modeling of the flare loops in still in its infancy. To begin with, one must satisfactorily deal with the complicated interplay between hydrodynamics, plasma physics, radiative transfer, and atomic and nuclear physics necessary to model the response of the atmosphere and the associated radiative signatures. The formidable complexity of this problem rules out analytic treatments (although with some drastic simplifications a crude analytic understanding of the qualitative physics taking place is attainable; Brown and Emslie, 1989; see Section 3). We are thus driven to the use of numerical computer codes. To date only approximate numerical treatments exist, and even these involve the use of sophisticated computational techniques and require many hours of CPU time on large machines. Even then, results from different codes can apparently yield substantially different results, even when attempting to solve the same 'benchmark' problem. These discrepancies can sometime be resolved through a realization that different numerical schemes (e.g., Lagrangian vs Eulerian) must of necessity handle a given problem in substantially different ways; however, there still remain differences that cannot readily be accounted for (see Kopp et al., 1986, for a discussion). At this stage, therefore, results from even single-fluid codes must be treated with some caution.

A further problem is that numerical codes, in order to represent a physical model of an actual flare, require the specification of not only initial and/or boundary conditions (see Section 3), but also the appropriate source terms in the momentum (Brown and Craig, 1984; McClymont and Canfield, 1984, Tamres, Canfield, and McClymont, 1986) and energy equations. However, the form of the flare energy input itself is not well known at this time, awaiting an understanding of the deep mysteries by which magnetic free energy is converted into accelerated particles and/or heat. Indeed, one of the major goals of flare loop modeling is to constrain the primary energy release mechanism through comparison of the predictions of candidate energy transport models with observation. A secondary, although equally challenging, goal is to understand the processes of energy transport themselves, using the flare as our 'laboratory' to analyze processes that are impossible to study in controlled terrestrial experiments.

Despite these somewhat disparaging remarks, however, a great deal of success has been achieved in explaining the observed characteristics of solar flares in terms of energization of a loop or series of loops. In the next section we shall examine the candidate energy transport mechanisms themselves, before discussing their application to flare loop modeling in subsequent sections. 


\section{Energy Transport Mechanisms}

Numerous mechanisms have been invoked to account for the spread of energy, generated by magnetic field dissipation in the primary energy release site, throughout the flare volume. They can be generally categorized into transport by particles, waves (including heat conduction), mass motions, and radiation.

\subsection{Particle Bombardment}

Prompt acceleration of both electrons and protons during the impulsive phase of a solar flare appears to be a common characteristic (see, e.g., Ramaty, 1986; Vlahos et al., 1986). Emslie (1983) has shown that both electrons and protons can contribute significantly to atmospheric heating, with protons characteristically heating at great depths (lower chromosphere and temperature minimum) and electrons predominating in the higher levels (corona and upper chromosphere). Due to the much lower energy per particle involved, electron heating involves a much greater particle flux and, hence, beam-associated electrical current. Thus, in determining the dynamics of the particles and the dissipation of their energy, we must consider not only 'single particle' processes, such as Coulomb collisions (Brown, 1972; Emslie, 1978) and magnetic field variation (Leach and Petrosian, 1981; Chandrashekar and Emslie, 1986), but also 'collective' processes, such as the driving of a beam-neutralizing return current (Knight and Sturrock, 1977; Emslie, 1980) and the possible generation of Langmuir and electroncyclotron waves (Vlahos and Papadopoulos, 1979; Emslie and Smith, 1984; McClements, 1987a, b). By contrast, in the case of proton bombardment only singleparticle processes need be considered, since the number of particles, and the associated electrical current, are relatively small. Note that in general energy transport by high energy particles is extremely rapid (see Section 4).

\subsection{MHD WAVES}

Because of the large ( $\gtrsim 10^{8} \mathrm{~cm} \mathrm{~s}^{-1}$ ) Alfvén velocities appropriate to flaring coronae, propagation of MHD waves represents another rapid a method of transporting energy throughout the flare volume. However, such waves are only significantly damped in regions of high electrical resistivity, and as such probably only play a significant role in the heating of the cool, partially ionized, lower layers of the atmosphere, around the temperature minimum region (Emslie and Sturrock, 1982). However, MHD waves propagating perpendicular to field lines may also represent an effective way of removing energy from the primary energy release region (see Spicer, Mariska, and Boris, 1986).

\subsection{Heat CONDUCTION}

Due to its strong dependence on temperature, heat conduction is a very effective mechanism of energy transport in the flaring corona (Emslie, 1985). By the same token, it is totally ineffective in the cool chromospheric layers, where radiation (Section 2.5) instead dominates (Emslie, Brown, and Machado, 1981). In the presence of a strong magnetic field, the heat flow is strongly anisotropic, transport parallel to the magnetic 
field being orders of magnitude more important than transport in the perpendicular direction. Although traditionally treated in the classical, diffusive, approach (Spitzer, 1962), the true processes of heat conduction in solar flares can be extremely complicated and varied. In regions of steep temperature gradient, the local temperature gradient is no longer, as in diffusive treatments, sufficient to determine the local heat flux; instead more global considerations, requiring a consideration of the variation of temperature over several mean free paths on either side of the region of interest, apply (e.g., Karpen and DeVore, 1987). The large heat fluxes associated with flare energization may approach the saturated heat flux limit (the maximum rate at which electrons with mean thermal speed $v_{e}$ can transport energy), so that the (free-streaming) flux $F \approx n v_{e}^{3}$ ( $v_{e}$ is the thermal velocity) becomes functionally dependent on the local density and temperature, rather than on the temperature and temperature gradient. In addition, the Onsager relations (e.g., Spitzer, 1962) imply that a heat flux has an associated electrical current. If driven to a suitably high level, the neutralizing return current (Knight and Sturrock, 1977) will excite plasma waves which in turn inhibit the conductive flux (see, e.g., Brown, Melrose, and Spicer, 1979; Smith and Lilliequist, 1979). Therefore, a full and proper treatment of conductive heat transfer in flares is a complex issue, requiring sophisticated numerical schemes to yield results which approach the true physical behavior of the plasma.

\subsection{MASS MOTIONS}

The steep pressure gradients established during the impulsive heating of preflare material are responsible for driving energetically significant bulk flows, as first pointed out by Craig and McClymont (1976). Indeed, all present-day models of flare loops (e.g., Pallavicini et al., 1983; Nagai and Emslie, 1984; Fisher, Canfield, and McClymont, 1985; MacNeice et al., 1984) have as their core a system of hydrodynamic equations. Driven sufficiently, such a system can develop hydrodynamic shocks, whose viscous dissipation can also be energetically significant. We shall return to a more detailed discussion of mass motions in flare loops in Sections 3 and 5.

\subsection{RADIATION}

Long recognized as a valuable diagnostic of flare energetics, it is now becoming increasingly apparent that the radiation field itself may contribute significantly to the energy budget of the flare, particularly in the optically thick lower layers of the atmosphere. Radiation 'backwarming' by soft X-ray radiation is an effective mechanism for heating the upper chromosphere (Somov, 1985; Machado, 1978), while EUV radiation produced in the transition region can be responsible for depositing significant energy around the temperature minimum (Emslie and Machado, 1979), as well as inducing charges in opacity that lead to enhanced absorption of photospheric radiation (Machado, Emslie, and Mauas, 1986). The complicated interplay between lines and continua of the plethora of atomic species to be found throughout a flare loop (from FeXXVI in the corona to neutral hydrogen and metals in the lower chromosphere) makes a through treatment of radiative energy balance exceedingly complicated, even in the quiet Sun (Vernazza, 
Avrett, and Loeser, 1981). Recently, however, preliminary models of radiative energy balance in flares, both empirical (Machado et al., 1980; Avrett, Machado, and Kurucz, 1986) and theoretical (McClymont and Canfield, 1983; Canfield, Fisher, and McClymont, 1983) have been put forward.

\section{Hydrodynamic Modeling of Flare Loops - Some General Comments}

The basic physics underlying the hydrodynamic response of the solar atmosphere to flare energy input is relatively straightforward (Brown and Emslie, 1989). The deposited energy first causes a temperature rise in the ambient material. Pressure gradients set up by this (generally nonuniform) heating then establish mass motions, as a result of which the density of the material changes. The subtle interplay between the energy input and output terms (which as we have seen in the preceding section, are functions of ambient parameters such as temperature, temperature gradient, and density) and the ambient conditions themselves renders a thorough understanding of the hydrodynamic response less than straightforward. The nature of the hydrodynamic equations themselves (a set of nonlinear coupled partial differential equations with integro-differential source terms) further adds to the complexity of the problem. Furthermore, the assumed initial and boundary conditions used in the definition of the problem in fact play a critical role in the evolution of the system. To see this, we note that the set of hydrodynamic equations generally possesses three sets of characteristics. One of these is stationary and corresponds to the local increase in temperature due to flare energy deposition. The other two are moving (one upward, one downward) and correspond to propagation of information, typically at a speed around the sound speed. Consider the downwardpropagating characteristics. At a given depth $z_{0}$, there is a corresponding time $\left(t_{0}\right.$, say) at which the first downward-moving characteristic from the top of the atmosphere arrives there. For times $t<t_{0}$, the specification of initial conditions for all $z$, plus information on the local heating, are clearly sufficient to prescribe the properties on all characteristics passing through $z=z_{0}$ and, hence, to determine the behavior of the gas there. However, for $t>t_{0}$, initialized information from the top boundary $(z=0)$ has already passed $z=z_{0}$, and the downward-propagating characteristic through $z_{0}$ thus originates at the top of the atmosphere (the $z=0$ plane) at a finite value of $t\left(t^{*}\right.$, say). Therefore, prescription of the characteristics through $z_{0}$ past time $t=t_{0}$ requires knowledge of the boundary conditions on $z=0$ up to time $t^{*}$. (For details of this argument, see Brown and Emslie, 1989.)

Most hydrodynamic simulations of flare loops provide this necessary boundary condition by considering one-half of a symmetrical loop, thereby effectively imposing the symmetry condition that the velocity vanish at the loop apex. However, such perfect symmetry is hardly likely to be realized in practice, and it would be a worthwhile exercise to consider in detail the effect that more realistic boundary conditions have on the solutions. 


\section{The Transition Region as a Diagnostic of Energy Transport Mechanisms}

Radiation from the chromosphere/corona region $\left(10^{4} \lesssim T \lesssim 10^{6} \mathrm{~K}\right)$ is dominated by optically thin UV lines. The intensity of such lines is given by the formula

$$
I=A \int n^{2} \frac{\mathrm{d} z}{\mathrm{~d} T} \mathrm{~d} T,
$$

where $A$ is a constant depending on the abundance of the responsible element relative to hydrogen and on atomic parameters relevant to the line in question. The quality $n^{2}(\mathrm{~d} z / \mathrm{d} T)$ is known as the Differential Emission Measure (DEM) and depends only on the structure of the atmosphere. Typically, as flare heating within a loop proceeds, the temperatures at which a given line is produced are realized at greater and greater depths (the transition region 'moves' downward), resulting in an enhancement of the $n^{2}$ factor. However, if the coronal part of the loop is also appreciably heated, then the larger conductive heat losses through the transition region necessitate a higher temperature gradient (and so lower $\mathrm{d} z / \mathrm{d} T$ ) at a given temperature. Thus the behavior of the DEM is a result of two competing factors. In a model in which the primary energy transport mechanism is thermal conduction from a hot corona, we would expect DEM and, therefore, the intensity in transition region UV lines, to decrease in response to flare energy input (Emslie and Nagai, 1985). Observations, however, show that UV line intensities are in fact well correlated with hard X-ray burst time profiles (Poland et al., 1984; Orwig and Woodgate, 1986). Since hard X-ray bursts are a good diagnostic of the energy input rate to the flare (e.g., Brown, 1971), we see that the assumption of conductively-dominated energetics is incompatible with the observations. On the other hand, Emslie and Nagai (1985) and Mariska and Poland (1985) have shown that the more spatially distributed heating corresponding to collisional degradation of nonthermal electron results is more modest decreases in $(\mathrm{d} z / \mathrm{d} T)$ coupled with substantial increases in $n^{2}$, so that the behavior of the DEM in such an energy transport model is indeed in accord with the hard X-ray/UV correlation observations. This success of the electron-heated hypothesis (or, more significantly, the failure of the thermal conduction hypothesis) leads us to consider electron-heated loop models in more detail in the next section.

\section{Soft X-Ray Diagnostics of Electron-Heated Loops}

One of the most powerful diagnostics of energy transport in flare loops is the shift and broadening of soft X-ray line profiles resulting from the flows set up by the flareassociated heating. Antonucci et al. (1982) have reported observations of broadening and blue asymmetry in the profile of the CaxIX resonance line at $3.177 \AA$ (' $w$ ', in the notation of Gabriel, 1972). They model this by a double gaussian fit to the line profile, the components of which are typically separated by a few $m \AA$, corresponding to a few hundred $\mathrm{km} \mathrm{s}^{-1}$ of upflow velocity. Emslie and Alexander (1987) used the electronheated hydrodynamic simulation results of Nagai and Emslie (1984) to synthesize 
Ca XIX ' $w$ ' profiles and found general agreement of both the absolute intensity and shape of the line with observation. (By contrast, Cheng, Karpen, and Doschek (1984) have modeled the response of a loop to a heat input with a gaussian spatial distribution. This heating function is ad hoc, is not based on any of the energy transport mechanisms discussed in Section 2, and in fact results in CaXIX ' $w$ ' profiles which disagree significantly with the observational data.) However, Emslie and Alexander found no evidence for a stationary component, as reported by Antonucci et al. (1982), and suggested that this may have been due to an error in the absolute calibration of the Solar Maximum Mission (SMM) Bent Crystal Spectrometer (BCS) instrument used. More recent work by McClements and Alexander (1989) has shown that there is no significant center-tolimb variation in the wavelength of the principal component, implying that this component is indeed stationary, contrary to the model results of Emslie and Alexander (1987). Mariska, Emslie, and Li (1989) and Li, Emslie, and Mariska (1989) note that Emslie and Alexander's loop length was sufficiently large that no significant accumulation of upwelling material at the apex of the loop occurred over the duration of the simulation, and suggested that the use of shorter loops would more naturally give rise to a stationary component at the loop apex. They consequently carried out a series of numerical models describing the hydrodynamic response of a loop to nonthermal electron input, varying the flux and spectrum of the injected electrons and computing the CaxIX ' $w$ ' line profiles for each model. Their results show clearly that, while blueshifted principal components do persist during the first few tens of seconds of energy input (in agreement with the modeling of Emslie and Alexander), a strong stationary component develops after about $30 \mathrm{~s}$ a time of order $L / c_{s}$, where $L$ is the half-length of the loop $\left(\approx 10^{9} \mathrm{~cm}\right)$ and $c_{s}$ the sound speed (roughly the velocity of the upward moving material). Averaged over the 30-50 s typical of the observations (Antonucci et al., 1982), the overall CaXIX ' $w$ ' profile, therefore, shows a principal component that corresponds to the strong emission late in the impulsive phase (and is therefore stationary), with the earlier blueshifts manifesting themselves only in a blue 'shoulder' on the averaged line profile. These time-integrated profiles agree very well with the observations, providing considerable support for the electron-heated model. In addition, the intensity of the blueshifted component of the CaXIX ' $w$ ' line correlates well (but nonlinearly) with the electron flux; together with hard X-ray bremsstrahlung observations, for which the intensity varies linearly with the total number of injected electrons, we can deduce the area over which the electrons are injected, a quantity not only of considerable interest to theoreticians, but also directly comparable with spatial images of the flare in, for example, the $\mathrm{H} \alpha$ line.

\section{Other Results Supporting Simple Loop Models}

In this section we briefly summarize results from other areas which also lend support to the simple electron-heated loop model concept. 


\subsection{THE EUV TO HARD X-RAY FLUX RATIO}

McClymont and Canfield (1986) have shown that, within a reasonable scatter, a simple (power-law) relationship exists between the fluxes in hard X-rays and broadband EUV (10-1030 ̊̊) from event to event. Such a relationship is in fact simply explained by a model in which all events correspond to the injection of nonthermal electrons into loops of the same area (of order $10^{16} \mathrm{~cm}^{2}$ ). In such a scenario, a doubling (say) of the electron flux produces a doubling of the hard $\mathrm{X}$-ray bremsstrahlung yield; it also overheats more of the chromosphere to coronal temperatures, so that a smaller fraction of the electron energy is deposited in cooler EUV-emitting layers. The observed EUV/hard X-ray flux relationship is consistent with heating by an electron beam with a power-law energy spectrum $E^{-\delta}$, with $\delta \approx 5$ (McClymont and Canfield, 1986). Furthermore, LaRosa and Emslie (1988) have shown that the large chromospheric beam fluxes implied by the model for large events require some form of a beam focusing (e.g., a convergence of the uiding magnetic field lines) to be present, so as to avoid embarrassingly high fluxes at the injection point, which would excite collective plasma instabilities and so saturate the bremsstrahlung yield at a level below that observed (see Emslie, 1980).

\subsection{Momentum Balance}

Zarro et al. (1988; see also Canfield et al., 1988) have shown that the upward momentum of the Caxix-emitting material (see Section 5) is in general balanced, to order of magnitude, by the downward momentum associated with the well-known redshift in $\mathrm{H} \alpha$ profiles (e.g., Švestka, 1976). This confirms that the blueshifted component of the CaXIX profile is produced by gas energized by heating of material in the upper atmosphere, such as by electron bombardment at the footpoints of a loop. (If the blueshifted material was instead associated with a mechanism which did not involve impulsive energy input, such as, for example, the rising of prominence material, then the momentum recoil would be absorbed by the whole Sun and would not have a clear observational signature.)

\subsection{EVOlution OF HARD X-RAY SPATIAL STRUCTURE}

In a simple electron-heated loop model, the hard X-rays are initially emitted predominantly from the dense chromospheric footpoints. Later, as a result of two factors, both of which are direct consequences of the collisional heating affected by the electron beam: (i) the evaporation of chromospheric material and (ii) the creation, through collisional heating, of a hot coronal source of thermal hard X-rays, the emission should become more diffusely distributed throughout the entire loop volume (Emslie, 1981b; Brown and Emslie, 1987). Results from the Hard X-Ray Imaging Spectrometer (HXIS) on SMM (e.g., Hoyng et al., 1981) show that this simple behavior indeed occurs; however, spatial resolution on the order of a few arc sec, together with temporal resolution of a few seconds, is necessary to trace the evolution of the structure from the initial double footpoint structure into the later diffuse source, and so test the relative roles of the factors above. Such instrumentation, using Fourier-transform spectrometers, is a major 
thrust of the Max '91 program for flare study at the next solar maximum (Dennis et al., 1988).

\section{4. $\mathrm{H} \propto$ PROFILES AND MORPHOLOGY}

Canfield, Gunkler, and Ricchiazzi (1984) report that the $\mathrm{H} \alpha$ profiles during the impulsive phase show broad wings which can only be satisfactorily explained by the Stark broadening associated with collisional electron heating of dense chromospheric layers to $\mathrm{H} \alpha$-emitting temperatures (see, however, Doschek et al., 1986). The fluxes inferred from modeling of the line profile are consistent with those required to produce the hard $\mathrm{X}$-ray burst (Canfield and Gayley, 1987). In addition, there is ample observational evidence showing that the brightenings occur at the feet of magnetic structures (e.g., Švestka, 1976; Tandberg-Hanssen and Emslie, 1988).

\subsection{IRON $\mathrm{K} \alpha$ EMISSION}

Tanaka, Watanabe, and Nitta (1984) and Emslie, Phillips, and Dennis (1985) have shown that impulsive enhancements in the iron $\mathrm{K} \alpha$ line (produced by inner-shell ionization of a near-neutral iron atom, follow by $2 p \rightarrow 1 s$ recombination into the innershell vacancy) occur simultaneously with hard X-ray bursts and that the intensity of the line is consistent with that expected from collisional ionization due to beamed nonthermal electrons in a simple loop geometry.

\section{Conclusions}

We have shown that, despite the obviously heuristic foundations on which the concept of a flare 'loop' is based, the modeling of such loops in fact yield results which are consistent with a large variety of observational data. This establishes on a more secure foundation two notions: namely (i) that magnetic loops are a fundamental constituent of solar flares, and (ii) that the primary energy release mechanism is an efficient particle accelerator. Evidently models that specifically invoke loop geometries (e.g., Spicer, 1977a, b; Colgate, 1978; Tajima, Brunel, and Sakai, 1982) should be more thoroughly pursued. We here also note recent observational evidence suggestive of energy release in loop-like structures. A detailed and systematic analysis of hard X-ray imaging compared with vector magnetograms demonstrated that, while many flares are triggered by the interaction of distinct flux systems, most of the energy released occurred within the loop of greatest shear (Machado et al., 1988). However, it must be re-emphasized that the models to date are clearly oversimplistic (e.g., assumed topology and geometry of the 'loop', treatment of the interrelationship between hydrodynamics and radiative transfer, etc.). Furthermore, the theoretical problem of how the required large flux of nonthermal electrons can be impulsively accelerated remains a formidable one (Heyvaerts, 1981; Vlahos et al., 1986). It, therefore, remains to be seen whether these loop models will survive the more intense scrutiny that will be provided by the stateof-the-art instrumentation now planned for the next solar maximum. 


\section{Acknowledgements}

I thank T. N. LaRosa for discussion and comments and J. C. Brown for clarifying the discussion of characteristics in Section 3. This work was supported by NASA Grants NAGW-294 and NAG5-500, and NSF Grants ATM-8715195 and AST-8351058.

\section{References}

Antonucci, E., Gabriel, A. H., Acton, L. W., Culhane, J. L., Doyle, J. G., Leibacher, J. W., Machado, M. E., Orwig, L. E., and Rapley, C. G.: 1987, Solar Phys. 78, 107.

Avrett, E. H., Machado, M. E., and Kurucz, R. L.: 1986, in D. F. Neidig (ed.), The Lower Atmosphere of Solar Flares, National Solar Observatory, p. 216.

Brown, D. G. and Emslie, A. G.: 1987, Solar Phys. 110, 305.

Brown, J. C.: 1971, Solar Phys. 18, 489

Brown, J. C.: 1972, Solar Phys. 26, 441.

Brown, J. C. and Craig, I. J. D.: 1984, Astron. Astrophys. 130, L5.

Brown, J. C. and Emslie, A. G.: 1989, Astrophys. J. (in press).

Brown, J. C., Melrose, D. B., and Spicer, D. S.: 1979, Astrophys. J. 228, 592.

Canfield, R. C. and Gayley, K. G.: 1987, Astrophys. J. 322, 999.

Canfield, R. C., Fisher, G. H., and McClymont, A. N.: 1983, Astrophys. J. 265, 507.

Canfield, R. C., Gunkler, T. A., and Ricchiazzi, P. J.: 1984, Astrophys. J. 282, 296.

Canfield, R. C., Zarro, D. M., Metcalf, T. R., and Lemen, J. R.: 1988, Bull. Am. Astron. Soc. $20,688$.

Chandrashekar, S. and Emslie, A. G.: 1986, Solar Phys. 107, 83.

Cheng, C.-C., Karpen, J. T., and Doschek, G. A.: 1984, Astrophys. J. 286, 787.

Colgate, S. A.: 1978, Astrophys. J. 221, 1068.

Craig, I. J. D. and McClymont, A. N.: 1976, Solar Phys. 50, 133.

Dennis, B. R. et al: 1988, Max '91 - Flare Research at the Next Solar Maximum, NASA.

Doschek, G. A. et al.: 1986, Energetic Phenomena on the Sun, NASA CP-2439, pp. 4-39.

Emslie, A. G.: 1978, Astrophys. J. 224, 241.

Emslie, A. G.: 1980, Astrophys. J. 235, 1055.

Emslie, A. G.: 1981a, Astrophys. Letters 22, 171.

Emslie, A. G.: 1981b, Astrophys. J. 245, 711.

Emslie, A. G.: 1983, Solar Phys. 84, 263.

Emslie, A. G.: 1985, Solar Phys. 98, 281.

Emslie, A. G. and Alexander, D.: 1987, Solar Phys. 110, 295.

Emslie, A. G. and Nagai, F.: 1985, Astrophys. J. 288, 779.

Emslie, A. G. and Smith, D. F.: 1984, Astrophys. J. 279, 882.

Emslie, A. G. and Sturrock, P. A.: 1982, Solar Phys. 80, 99.

Emslie, A. G., Brown, J. C., and Machado, M. E.: 1981, Astrophys. J. 246, 337.

Emslie, A. G., Phillips, K. J. H., and Dennis, B. R.: 1985, Solar Phys. 103, 89.

Fisher, G. H., Canfield, R. C., and McClymont, A. N.: 1985, Astrophys. J. 289, 414.

Gabriel, A. H.: 1972, Monthly Notices Roy. Astron. Soc. 160, 99.

Heyvaerts, J.: 1981, in E. R. Priest (ed.), Solar Flare Magnetohydrodynamics, Gordon and Breach Publ. Co., New York.

Hoyng, P. et al:: 1981, Astrophys. J. 246, L155.

Karpen, J. T. and DeVore, C. R.: 1987, Astrophys. J. 320, 904.

Knight, J. W. and Sturrock, P. A.: 1977, Astrophys. J. 218, 306.

Kopp, R. A., Fisher, G. H., MacNeice, P., McWhirter, R. W. P., and Peres, G.: 1986, Energetic Phenomena on the Sun, NASA CP-2439, Chapter 7.

LaRosa, T. N. and Emslie, A. G.: 1988, Astrophys. J. 326, 997.

Leach, J. and Petrosian, V.: 1981, Astrophys. J. 251, 781.

Li, P., Emslie, A. G., and Mariska, J. T.: 1989, Astrophys. J. (in press).

Machado, M. E.: 1978, Solar Phys. 60, 341. 
Machado, M. E., Emslie, A. G., and Mauas, P. J.: 1986, Astron. Astrophys. 159, 33.

Machado, M. E., Avrett, E. H., Vernazza, J. E., and Noyes, R. W.: 1980, Astrophys. J. $242,336$.

Machado, M. E., Moore, R. L., Hernandez, A. M., Rovira, M. G., Hagyard, M. J., and Smith, J. B., Jr.: 1988, Astrophys. J. 326, 425.

MacNeice, P., McWhirter, R. W. P., Spicer, D. S., and Burgess, A.: 1984, Solar Phys. 90, 357.

Mariska, J. T. and Poland, A. I.: 1985, Solar Phys. 96, 317.

Mariska, J. T., Emslie, A. G., and Li, P.: 1989, Astrophys. $J$. (in press).

McClements, K. G.: 1987a, Astron. Astrophys. 175, 255.

McClements, K. G.: 1987b, Solar Phys. 109, 355.

McClements, K. G. and Alexander, D.: 1989, Solar Phys. (submitted).

McClymont, A. N. and Canfield, R. C.: 1983, Astrophys. J. 265, 483.

McClymont, A. N. and Canfield, R. C.: 1984, Astron. Astrophys. 136, L1.

McClymont, A. N. and Canfield, R. C.: 1986, Astrophys. J. 305, 936.

Melrose, D. B. and Dulk, G. A.: 1982, Astrophys. J. 259, 844.

Melrose, D. B. and Dulk, G. A.: 1984, Astrophys. J. 282, 308.

Nagai, F. and Emslie, A. G.: 1984, Astrophys. J. 279, 896.

Orwig, L. E. and Woodgate, B. E.: 1986, in D. F. Neidig (ed.), The Lower Atmosphere of Solar Flares, National Solar Observatory, p. 306.

Pallavicini, R., Peres, G., Serio, S., Vaiana, G., Acton, L., Leibacher, J., and Rosner, R.: 1983, Astrophys. J. $270,270$.

Poland, A. I., Orwig, L. E., Mariska, J. T., Nakatsuka, R., and Auer, L. H.: 1984, Astrophys. J. $280,457$.

Ramaty, R.: 1986, in P. A. Sturrock (ed.), Physics of the Sun, Vol. II, D. Reidel Publ. Co., Dordrecht, Holland, p. 291 .

Shoub, E. C.: 1983, Astrophys. J. 266, 339.

Smith, D. F. and Lilliequist, C. G.: 1979, Astrophys. J. 232, 582.

Somov, B. V.: 1975, Solar Phys. 42, 235.

Spicer, D. S.: 1977a, NRL Report 8036.

Spicer, D. S.: 1977b, Solar Phys. 53, 305.

Spicer, D. S., Mariska, J. T., and Boris, J. P.: 1986, in P. A. Sturrock (ed.), Physics of the Sun, Vol. II, D. Reidel Publ. Co., Dodrecht, Holland, p. 181.

Spitzer, L. W., Jr.: 1962, Physics of Fully Ionized Gases, Intergraph.

Svestka, Z.: 1976, Solar Flares, D. Reidel Publ. Co., Dordrecht, Holland.

Tajima, T., Brunel, F., and Sakai, J.: 1982, Astrophys. J. 258, L45.

Tamres, D. H., Canfield, R. C., and McClymont, A. N.: 1986, Astrophys. J. 309, 409.

Tanaka, K., Watanabe, T., and Nitta, N.: 1984, Astrophys. J. 282, 793.

Tandberg-Hanssen, E. and Emslie, A. G.: 1988, The Physics of Solar Flares, Cambridge University Press, Cambridge.

Vernazza, J. E., Avrett, E. H., and Loeser, R.: 1981, Astrophys. J. Suppl. 45, 635.

Vlahos, L. and Papadopoulos, K.: 1979, Astrophys. J. 233, 717.

Vlahos, L. et al.: 1986, in M. R. Kundu and B. E. Woodgate (eds.), Energetic Phenomena on the Sun, NASA CP-2439.

Zarro, D., Canfield, R. C., Strong, K. T., and Metcalf, T. R.: 1988, Astrophys. J. 324, 582. 